The Astrophysical Journal, 347:195-200, 1989 December 1

(C) 1989. The American Astronomical Society. All rights reserved. Printed in U.S.A.

\title{
ON THE METAL ABUNDANCE OF LOW-ACTIVITY GALACTIC NUCLEI
}

\author{
Thaisa Storchi BergmanN ${ }^{1}$ and Miriani Griselda Pastoriza ${ }^{1}$ \\ Instituto de Fisica, Universidade Federal do Rio Grande do Sul \\ Received 1988 March 30; accepted 1989 June 2
}

\begin{abstract}
The characteristics of the nuclear spectrum of six Seyfert 2 and LINER nuclei with low activity and strong lines of ionized nitrogen are investigated. From the equivalent widths of the absorption lines, the age and metallicity of the stellar population are derived. A comparison of the observed emission-line intensities with photoionization and shock model calculations leads to the conclusion that these nuclei are photoionized by a power-law continuum, and that the only way to reproduce the large $[\mathrm{N} \mathrm{II}] / \mathrm{H} \alpha$ ratio for at least four of them is with an overabundance of nitrogen relative to the other heavy elements.

Subject headings: galaxies: abundances — galaxies: stellar content
\end{abstract}

\section{INTRODUCTION}

Previous works, such as those of Burbidge and Burbidge $(1962,1965)$, have shown that the emission spectrum of the nucleus of early-type galaxies frequently presents the [N II] $\lambda 6584$ line stronger than $\mathrm{H} \alpha$. This relation is observed in several Seyfert 2 galaxies and is one of the most conspicuous characteristics of the spectrum of LINERs (Heckman 1980a, $b$; Stauffer $1982 a, b$; Keel $1983 a, b$ ).

Although it has been concluded that the enhancement of $[\mathrm{N}$ II $] / \mathrm{H} \alpha$ is largely an excitation effect (Pagel 1983), there are some nuclei, such as that of M51, which present higher $[\mathrm{N} \mathrm{II}] / \mathrm{H} \alpha$ ratios than can be accounted for by shock or powerlaw photoionization with solar abundance (Rose and Searle 1982).

In order to investigate the reason for a high $[\mathrm{N}$ II $] / \mathrm{H} \alpha$ ratio, we have selected six low-activity nuclei which show $[\mathrm{N} \mathrm{II}] / \mathrm{H} \alpha$ comparable to the M51 value and have analyzed their spectra in detail.

It is interesting to note that among the selected galaxies are three of the nearest with Seyfert 2 nuclei, in agreement with the result that a high $[\mathrm{N} \mathrm{II}] / \mathrm{H} \alpha$ ratio is found preferentially in nearby galaxies because it is a characteristic of the very nucleus (Rubin and Ford 1986). If one could observe in the more distant galaxies a region as small as is observed for the nearby ones, the number of galaxies presenting high $[\mathrm{N}$ II $] / \mathrm{H} \alpha$ ratios would probably be much larger than that is known today.

All the selected galaxies present strong absorption lines which we have used to study the stellar population of the nuclei. We have then subtracted a representative template for the absorption spectrum and have analyzed the emission component, using several photoionization and shock models.

\section{THE SAMPLE}

The selected galaxies are NGC 1358, NGC 1386, NGC 4941, and NGC 6300, all with Seyfert 2 nuclei, NGC 3312, which is a LINER, and NGC 7743, an intermediate case between a Seyfert 2 and a LINER (Phillips, Charles, and Baldwin 1983; Stauffer $1982 a, b$ ). The morphological types and the apparent and corrected absolute magnitudes and distances (Sandage and Tammann 1981) are listed in Table 1. The last column

\footnotetext{
${ }^{1}$ Visiting Observer, Cerro Tololo Inter-American Observatory, National Optical Astronomy Observatories.
}

shows the linear dimension subtended at the galaxy by the slit width used in the observations.

\section{OBSER VATIONS}

The observations were made with the $1 \mathrm{~m}$ telescope of Cerro Tololo Inter-American Observatory using the Cassegrain spectrograph and 2D-Frutti two-dimensional photon counting detector, originally developed by S. Schectman (Colbeck 1987). NGC 1358, NGC 1386, NGC 6300, and NGC 7743 were observed in 1986 September with a slit width of 8", NGC 3312 and NGC 4941 were observed in 1987 February with a slit width of $5^{\prime \prime}$. The spectral region covered was $3600-7000 \AA$, with $5 \AA$ resolution at $5000 \AA$ with both slits (as measured from the full width at half-maximum of comparison lamp lines). All the spectra were flux calibrated using standard stars from the list of Stone and Baldwin (1983).

The spectra have been corrected for galactic absorption (Sandage and Tammann 1981) and internal absorption due to the inclination of the disk of each galaxy using the mean relation for each morphological type between color excess $E(B-V)$ owing to inclination effects and axial ratio $b / a$ according to Figure 8 of Bica and Alloin (1986). We have used the interstellar reddening curve as parametrized by Seaton (1979).

\section{STELLAR POPULATION}

In order to study the stellar population of the nuclei, we have measured the equivalent widths $(W)$ of the following absorption lines: $K(\mathrm{Ca}$ II $), \mathrm{CN}(\lambda 4125), G$ band, $\mathrm{Mg} \mathrm{I}+\mathrm{Mg} \mathrm{H}$, and the Balmer lines $\mathbf{H} \delta, \mathbf{H} \gamma$, and $\mathbf{H} \beta$. The corresponding adopted spectral windows are 3908-3952 $\AA, 4150-4214 \AA$, 4284-4318 $\AA, 5156-5196 \AA, 4082-4124 \AA, 4318-4364 \AA$, and 4846-4884 $\AA$. Table 2 shows the measured values, together with the continuum fluxes at $4020 \AA$ and $4570 \AA$ relative to the flux at $5870 \AA$. The mean errors range from $10 \%$ to $20 \%$ for $W$ larger than $5 \AA$, and from $30 \%$ to $40 \%$ for smaller $W$.

Bica (1988) has grouped nuclear spectra of Shapley-Ames galaxies according to similar stellar population based on equivalent widths of spectral features. Each group was synthesized in terms of components in the age-metallicity plane using a base of observed star cluster spectra, defining in this way the templates. In this paper, we use the templates representative of the population in the nuclei of spiral galaxies. The importance of using these templates is to avoid the contamination of emis- 
TABLE 1

BASIC DATA

\begin{tabular}{clcccc}
\hline \hline Galaxy & Morphology & $\boldsymbol{B}_{\boldsymbol{T}}$ & $\begin{array}{c}\text { Distance } \\
(\mathbf{M p c})\end{array}$ & $\boldsymbol{M}_{\boldsymbol{B}}$ & $\begin{array}{c}\text { Slit } \\
(\mathrm{kpc})\end{array}$ \\
\hline NGC $1358 \ldots \ldots$ & SBa(s)I & 13.0 & 82.4 & -21.6 & 3.2 \\
NGC $1386 \ldots \ldots$ & Sa & 12.0 & 30.8 & -21.4 & 1.2 \\
NGC $3312 \ldots \ldots$ & Sab(r) & 12.7 & 49.9 & -21.9 & 1.2 \\
NGC $4941 \ldots \ldots$ & Sab(s)II & 11.9 & 17.5 & -20.1 & 0.4 \\
NGC $6300 \ldots \ldots$ & SB(rs) $\mathrm{b}^{\mathrm{a}}$ & $11.0^{\mathrm{a}}$ & 18.0 & -21.3 & 0.7 \\
NGC $7743 \ldots \ldots$ & SBa & 12.1 & 40.6 & -21.0 & 1.6 \\
\hline
\end{tabular}

${ }^{\text {a }}$ Buta 1987.

sion lines, which are normally present in the spectra of spiral galaxies. Our measured equivalent widths were then compared with those for the templates, and, for each of the selected nuclei, it was possible to find one template with $W$ values within $10 \%$ of the measured ones or two templates such that the measured values were intermediate between those of the two templates. Due to the fact that the spectral resolution of the templates is $10 \AA$, we have smoothed our data to the same resolution before the measurements of the equivalent widths.

Based on Bica's results from the stellar population synthesis, we have derived the following characteristics for the stellar population of the selected nuclei: percentage contribution of very old stars $\left(\sim 10^{10} \mathrm{yr}\right)$, of intermediate-age stars $\left(10^{9}\right.$ to $5 \times 10^{9} \mathrm{yr}$ ), and of stars younger than $5 \times 10^{8} \mathrm{yr}$ to the total light at $5870 \AA$. We have also obtained the maximum metallicity reached by the last generation of stars. Table 3 lists the corresponding values, together with the reddening $E(B-V)$ necessary to make the observed continuum match that of the selected template.

From Table 3 we can see that, for all nuclei, more than $80 \%$ of the light at $5870 \AA$ comes from the very old stars. Also, an intermediate-age component contributing $10 \%$ to $15 \%$ is always present. For NGC 1358, NGC 3312, and NGC 4941, these are the only components, but for NGC 1386, NGC 6300, and NGC 7743, there is a small contribution from young stars.
We can also conclude that the metallicity of the nuclei ranges from solar to 4 times solar.

Figure 1 shows, for each galaxy, the observed spectrum, the selected stellar population template, and the difference between them, where only the emission component is left.

\section{THE EMISSION-LINE SPECTRUM}

After subtraction of the stellar component, we have measured the emission lines, using Gaussian fits to the profiles. Table 4 shows integrated fluxes relative to [O III] $\lambda 5007$. Also given is the absolute flux in this line and the full widths at half-maximum (FWHM) for [O III] $\lambda 5007$ and [N II] $\lambda 6584$. The FWHM values have been approximately corrected for the instrumental profile by assuming the relation (observed $\mathrm{FWHM}^{2}=$ (intrinsic FWHM $^{2}+$ (instrumental FWHM $^{2}$.

\section{a) Line Profiles}

All line profiles are broadened relative to the instrumental profile. NGC 1358, NGC 4941, NGC 6300, and NGC 7743 have gas velocity dispersions in the range $300-400 \mathrm{~km} \mathrm{~s}^{-1}$, while NGC 1386 and NGC 3312 present larger values. It would be interesting to observe these galaxies with higher resolution. For NGC 1386 we have an spectrum with $3 \AA$ resolution of the $[\mathrm{O}$ III] $\lambda \lambda 4959+5007$ region, shown in Figure 2. Two components are necessary to reproduce the profiles, one with FWHM of $520 \mathrm{~km} \mathrm{~s}^{-1}$ and other with FWHM equal to the instrumental value $\left(160 \mathrm{~km} \mathrm{~s}^{-1}\right)$. The broad component is displaced $162 \mathrm{~km} \mathrm{~s}^{-1}$ to the red relative to the narrow component, which is at rest relative to the absorption lines, indicating a radial motion of the broad-line gas relative to the galaxy.

\section{b) Comparison with Photoionization Models}

In order to study the physical conditions and chemical composition of the nuclear gas we have compared the following emission-line ratios with values obtained from model calculations: $[\mathrm{N}$ II $](\lambda \lambda 6548+6584) / \mathrm{H} \alpha,[\mathrm{S}$ II $](\lambda \lambda 6717+6731) / \mathrm{H} \alpha$,

TABLE 2

Equivalent Widths of ABsorption Lines and ContinuUm FluXes

\begin{tabular}{|c|c|c|c|c|c|c|c|c|c|}
\hline Galaxy & $K$ & $\mathrm{CN}$ & $G$ band & $\mathrm{Mg}$ & $\mathbf{H} \delta$ & $\mathrm{H}$ & $\mathbf{H} \beta$ & $\lambda 4020^{\mathrm{a}}$ & $\lambda 4570^{\mathrm{a}}$ \\
\hline NGC $1358 .$. & 17.0 & 11.7 & 10.0 & 9.0 & 2.5 & $2.8 \mathrm{e}$ & $1.0 \mathrm{e}$ & 0.44 & 0.73 \\
\hline NGC $1386 \ldots$ & 13.2 & 10.2 & 8.9 & 7.1 & 5.1 & 5.1 & $2.8 \mathrm{e}$ & 0.63 & 0.84 \\
\hline NGC $3312 \ldots$ & 18.3 & 10.5 & 9.5 & 8.2 & 3.9 & 4.1 & $2.9 \mathrm{e}$ & 0.44 & 0.77 \\
\hline NGC 4941 . & 17.2 & 12.1 & 10.4 & 7.2 & 4.0 & $3.4 \mathrm{e}$ & $2.9 \mathrm{e}$ & 0.64 & 0.94 \\
\hline NGC $6300 \ldots \ldots \ldots \ldots \ldots \ldots$ & 13.0 & 10.5 & 9.1 & 6.5 & $3.1 \mathrm{e}$ & 5.6 & 4.3 & 0.67 & 0.95 \\
\hline NGC $7743 \ldots \ldots \ldots \ldots \ldots \ldots$ & 13.4 & 10.6 & 8.0 & 5.8 & 7.4 & 8.0 & 5.8 & 0.61 & 0.85 \\
\hline
\end{tabular}

NoTE. - e contaminated by emission.

a Continuum flux relative to the value at $5870 \AA$.

TABLE 3

Percent Contribution of Populations of Different Ages, Maximum Metallicity and Internal REDDENING OF THE NUCLEI

\begin{tabular}{|c|c|c|c|c|c|}
\hline Galaxy & $10^{10} \mathrm{yr}$ & $10^{9}+5 \times 10^{9} \mathrm{yr}$ & $<5 \times 10^{8} \mathrm{yr}$ & $\log \left(Z / Z_{0}\right)$ & $E(B-V)$ \\
\hline NGC $1358 \ldots \ldots \ldots \ldots \ldots \ldots$ & 88 & 12 & ... & 0.6 & 0.12 \\
\hline NGC $1386 \ldots \ldots \ldots \ldots \ldots \ldots$ & 84 & 13 & 3 & 0.3 & 0.05 \\
\hline NGC $3312 \ldots \ldots \ldots \ldots \ldots \ldots$ & 88 & 12 & $\ldots$ & 0.3 & 0.05 \\
\hline NGC $4941 \ldots \ldots \ldots \ldots \ldots \ldots$ & 87 & 13 & $\ldots$ & 0.3 & 0.10 \\
\hline NGC $6300 \ldots \ldots \ldots \ldots \ldots \ldots$ & 84 & 12 & 4 & 0.3 & $\ldots$ \\
\hline NGC $7743 \ldots \ldots \ldots \ldots \ldots . . . .$. & 82 & 12 & 6 & 0.0 & 0.05 \\
\hline
\end{tabular}



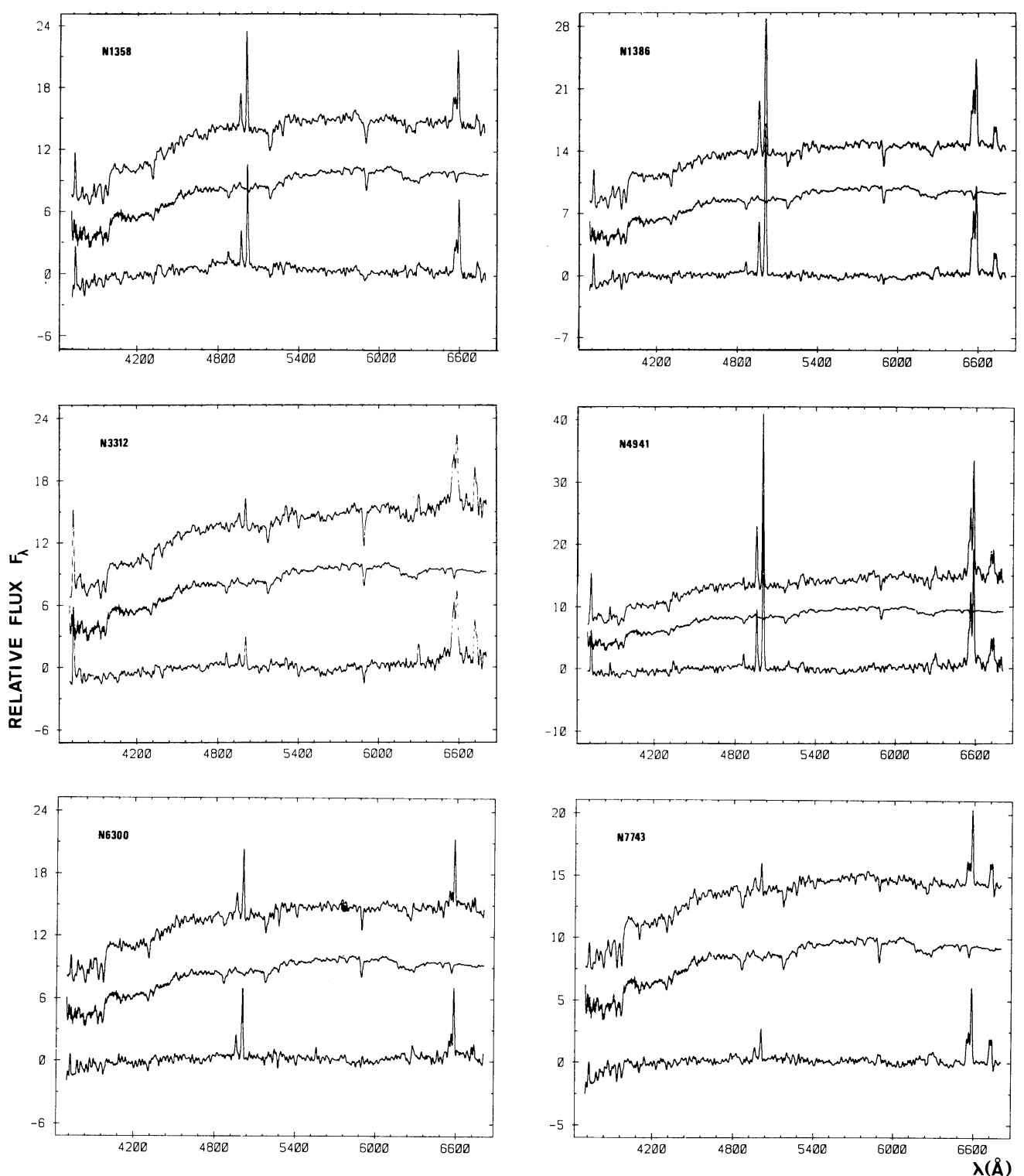

Fig. 1.-From top to bottom, the observed spectrum, the selected template, and their difference

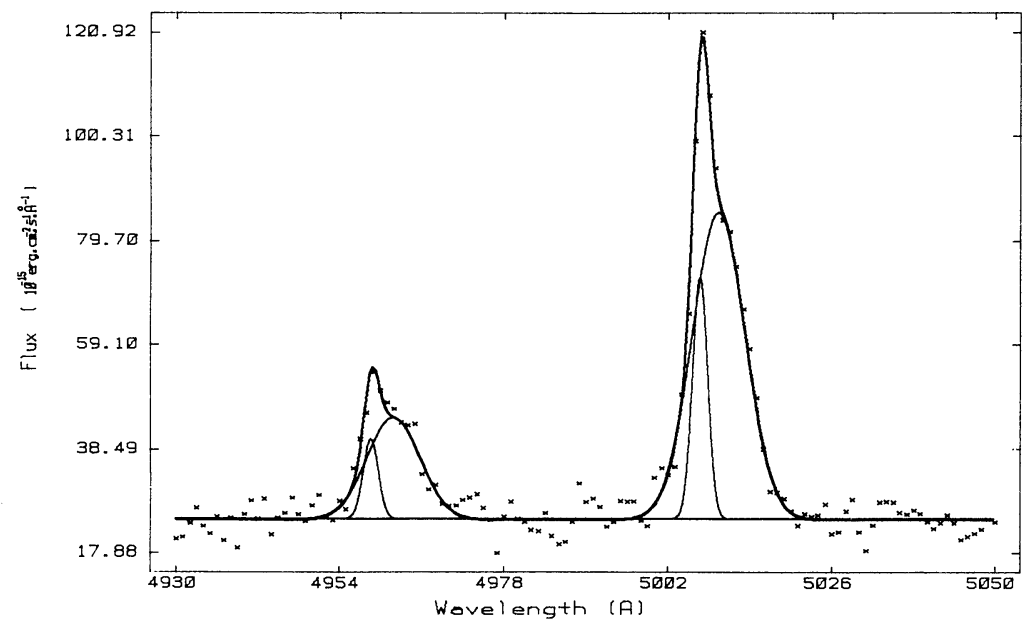

FIG. 2. - $[\mathrm{O}$ III $] \lambda \lambda 4959+5007$ profiles for NGC 1386. Crosses represent observational points; thin lines, the individual Gaussians fitted; thick line, the resulting profile. 
TABLE 4

EMISSION-LINE INTENSITIES

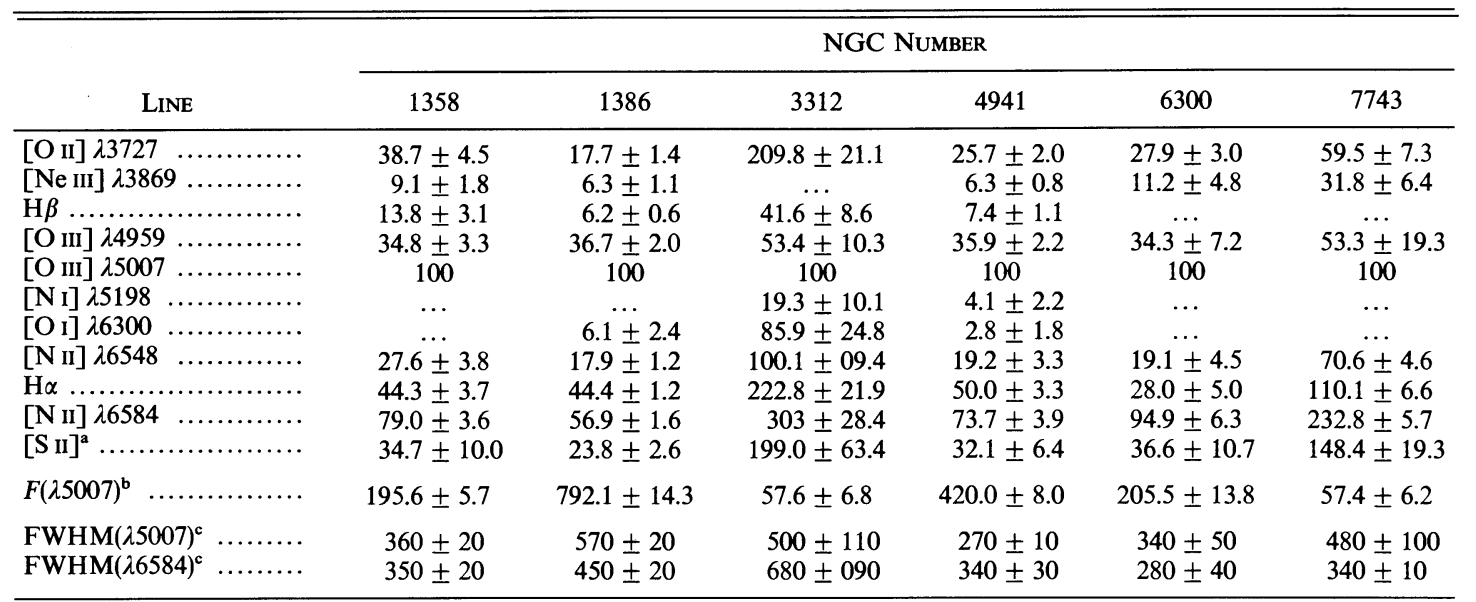

a Sum of $\lambda 6717$ and $\lambda 6731$.

b Observed flux in units of $10^{-15} \mathrm{ergs} \mathrm{cm}^{-2} \mathrm{~s}^{-1}$.

c Full width at half-maximum in $\mathrm{km} \mathrm{s}^{-1}$.

$\left[\begin{array}{ll}\mathrm{N} & \mathrm{II}\end{array}\right] /\left[\begin{array}{ll}\mathrm{S} & \mathrm{II}\end{array}\right],\left[\begin{array}{ll}\mathrm{O} & \mathrm{II}\end{array}\right](\lambda \lambda 3726+3729) /\left[\begin{array}{ll}\mathrm{O} & \mathrm{III}\end{array}\right]$, and $\left[\begin{array}{ll}\mathrm{N} & \mathrm{II}\end{array}\right] /$ ([O II $]+\left[\begin{array}{ll}\mathrm{O} & \mathrm{III}\end{array}\right]$ ). All these ratios have been corrected by reddening assuming that a value of $\mathrm{H} \alpha / \mathrm{H} \beta$ larger than the case $B$ recombination value is due to internal absorption. Nevertheless, only the last two ratios are affected by reddening, and the main results of this work do not depend on its value.

First we have used the photoionization code CLOUDY (Ferland and Truran 1980; Ferland and Netzer 1983) for constant density nebulae photoionized by a nonthermal continuum having the form:

$$
f_{v}=v^{\alpha} \exp \left(-v / v_{\text {high cut }}\right) \exp \left(-v_{\text {low cut }} / v\right) \text {. }
$$

The models which better represent the observed values have the following parameters: $\alpha=-1.5, v_{\text {high cut }}=10^{10} \operatorname{Ry}\left(\sim 10^{11}\right.$ $\mathrm{eV}$ ), and $v_{\text {low cut }}=0 \mathrm{eV}$. Since the observed relative intensities of the [S II] lines $I(6731) / I(6717)$ is $\sim 1$ (Fig. 1), and the critical

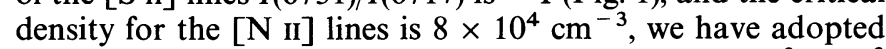
two representative values for the gas density: $N_{e}=10^{3} \mathrm{~cm}^{-3}$ and $N_{e}=10^{4} \mathrm{~cm}^{-3}$. The ionization parameter was restricted to $10^{-3.5}<U<10^{-3}$ for $N_{e}=10^{4} \mathrm{~cm}^{-3}$ and $10^{-3}<U<10^{-2.5}$ for $N_{e}=10^{3} \mathrm{~cm}^{-3}$ in order to reproduce the observed $[\mathrm{O}$ II $] /\left[\begin{array}{lll}\mathrm{O} & \mathrm{III}\end{array}\right]$ ratio. The corresponding total hydrogen column densities are $n_{c}=2.4 \times 10^{20} \mathrm{~cm}^{-2}$ for $N_{e}=$ $10^{4} \mathrm{~cm}^{-3}$ and $U=10^{-3.5} ; n_{c}=7.5 \times 10^{20} \mathrm{~cm}^{-2}$ for the same $N_{e}$ and $U=10^{-3}$; and $n_{c}=3.8 \times 10^{20} \mathrm{~cm}^{-2}$ for $N_{e}=10^{3}$ $\mathrm{cm}^{-3}$ and $U=10^{-3}$.

Figure 3 shows four diagrams involving intensity ratios of the strongest emission lines observed in the spectra. The diagrams present the observed values, together with the model values which best reproduce them. As a reference, we have also plotted the intensity ratios observed in the nucleus of M51 (Rose and Searle 1982), M81 (Peimbert and Torres-Peimbert 1981), and M87 (Stauffer and Spinrad 1979), which also show high $[\mathrm{N}$ II $] / \mathrm{H} \alpha$ ratios.

We have verified that, for solar abundance models, a change in $U$ and $N_{e}$ produces essentially an horizontal displacement in the diagrams, that is, only a change in the $[\mathrm{O} \mathrm{II}] /[\mathrm{O} \mathrm{III}]$ ratio. The ratios $\left[\begin{array}{ll}\mathrm{N} & \mathrm{II}\end{array}\right] / \mathrm{H} \alpha,\left[\begin{array}{ll}\mathrm{N} & \mathrm{II}\end{array}\right] /\left(\left[\begin{array}{ll}\mathrm{O} & \mathrm{II}\end{array}\right]+\left[\begin{array}{ll}\mathrm{O} & \mathrm{III}\end{array}\right]\right),\left[\begin{array}{ll}\mathrm{S} & \mathrm{II}\end{array}\right] / \mathrm{H} \alpha$, $[\mathrm{N}$ II $] /[\mathrm{S}$ II $]$ are practically insensitive to this change. We have also investigated the effect of varying the spectral index: for $\alpha=-1$, with cutoff at $100 \mathrm{Ry}$, the $[\mathrm{N}$ II $] / \mathrm{H} \alpha$ values are slightly higher than for $\alpha=-1.5$, but not enough to reproduce the observed ones. The $[\mathrm{N} \mathrm{II}] /([\mathrm{O}$ II $]+[\mathrm{O}$ III $])$ ratio for $\alpha=-1$ is almost the same as that for $\alpha=-1.5$. In order to try to reproduce the high observed [N II] intensities, we enhanced the abundances of all the heavy elements by a factor of 2 : the result is a very small increase in $[\mathrm{N}$ II $] / \mathrm{H} \alpha$ and no change in $[\mathrm{N} \mathrm{II}] /([\mathrm{O} \mathrm{II}]+[\mathrm{O} \mathrm{III}])$. This suggests that, in order to reproduce the observed ratios, nitrogen should be overabundant relative to oxygen. We have then computed models with nitrogen 3, 5, and 10 times solar and solar abundance for the other elements which are shown in Figures $3 a-3 d$. From the figures, it can be seen that the galaxies NGC 1358, NGC 6300, NGC 7743, M81, and M87 have nitrogen abundance 3 times solar; M51, 5 times solar; NGC $3312 \sim 2$ times solar; NGC 1386 and NGC 4941 have a nitrogen abundance between solar and 2 times solar. An overabundance of nitrogen has been also previously found for M51, M81, and M87 (Rose and Searle 1982; Peimbert and Torres-Peimbert 1981; Stauffer and Spinrad 1979).

All the galaxies in our sample show $[\mathrm{S}$ II $] / \mathrm{H} \alpha$ higher than calculated in the solar abundance models (line in Fig. 3c) and $[\mathrm{N} \mathrm{II}] /[\mathrm{S} \mathrm{II}]$ about equal to the solar abundance case (Fig. $3 d$ ). In order to understand this apparent contradiction, we have calculated two models with sulfur overabundance of 2 and 5 times solar plotted in Fig. $3 c$ as open circles. It can be seen that these models can reproduce the observed values. indicating that sulfur could be also overabundant.

We have obtained similar results, concerning the nitrogen abundance, using the photoionization code of Gruenwald and Pequignot (1987) for the same range of input parameters as used for CLOUDY. We also tested an integrated model (Viegas-Aldrovandi and Gruenwald 1988) considering the contribution of clouds of different densities following a distribution law $f(n) \propto n^{-b}$ for $10^{2}<n<10^{6} \mathrm{~cm}^{-3}$ with a blackbody of $10^{5} \mathrm{~K}$ as the ionizing source. The models which best reproduce the observations have $b=3$, which means that the lowdensity clouds dominate the contribution to the line fluxes. This last model, with nitrogen abundance solar and 2 times solar, is also plotted in the diagrams as filled squares. One interesting aspect of this model is that it can reproduce the 

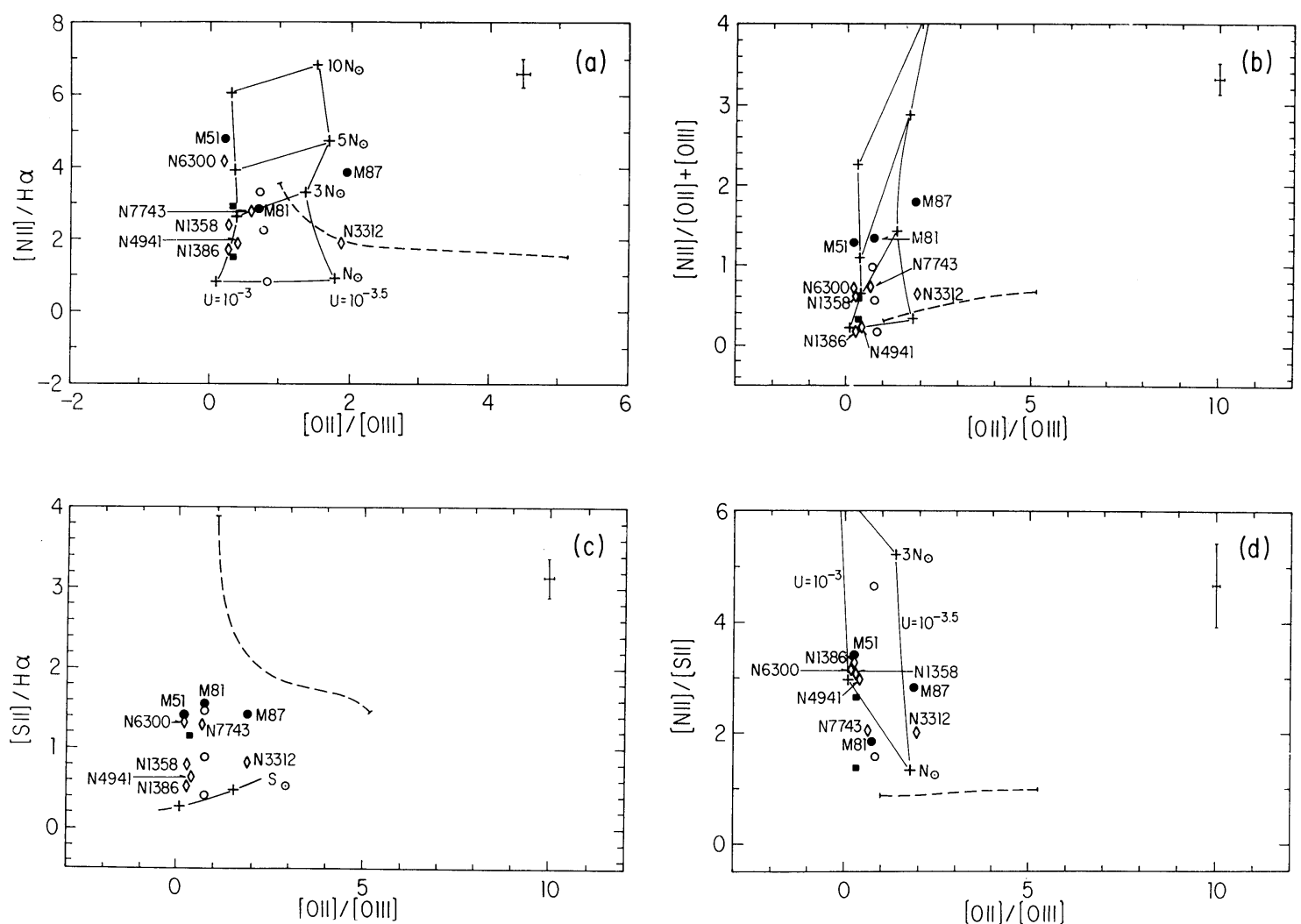

FIG. 3. $-(a)-(d)$ Emission-line ratios: (open losengles) observed values; ( filled circles) reference galaxies, (crosses) Ferland photoionization models with $\alpha=1.5$, $N_{e}=10^{4} \mathrm{~cm}^{-3}, U=10^{-3}$ (left), $U=10^{-3.5}$ (right). Solar abundances for all elements except nitrogen: solar (bottom), 3 times solar, 5 and 10 times solar (top); (open circles) Ferland models with $U=10^{-3}$ and $N_{e}=10^{3} \mathrm{~cm}$; (filled squares) integrated photoionization models with solar (bottom) and 2 times solar abundance of nitrogen (top); (dashed line) shock wave integrated models from low-velocity dominating clouds (left) to high-velocity dominating clouds (right). (c) Points for sulfur abundance: solar, 2 times solar, and 5 times solar (open circles). Average error bars are shown in upper right corners.

observed values of $[\mathrm{S} \mathrm{II}] / \mathrm{H} \alpha$ with solar abundance of $\mathrm{S} / \mathrm{H}$ and the observed $[\mathrm{N}$ II $] /[\mathrm{S}$ II $]$ with $\mathrm{N} / \mathrm{H} 2-3$ times solar. This suggests that in these nuclei the observed $[\mathrm{S} \mathrm{II}] / \mathrm{H} \alpha$ could be due to the presence of a distribution of clouds of different densities rather than an overabundance of sulfur. In fact, a wide range of electron densities has been previously found in other active galactic nuclei (Filippenko and Sargent 1988; Filippenko and Halpern 1984). Also, for NGC 1386, we found that the [O III] emission-line region is composed of two different zones $(\S \mathrm{Vc})$.

Integrated shock models assuming the contribution of clouds of different velocities following the distribution $f(v) \propto v^{-b}$ for $100<v<600 \mathrm{~km} \mathrm{~s}^{-1}$ were also investigated (Viegas-Aldrovandi 1988). Figures $3 a-3 d$ also show these models for $-10<b<10$ (dashed lines) where it can be seen that they give $[\mathrm{N}$ II $] / \mathrm{H} \alpha$ ratios near the observed ones when low-velocity clouds dominate $(b=10)$ but fail to reproduce all the other observed ratios. We also checked the results of composite models, taking into account both shocks and photoionization (Viegas-Aldrovandi and Contini 1988) and have found that the models with $[\mathrm{O} \mathrm{II}] /[\mathrm{O} \mathrm{III}]$ ratio similar to the observed ones have $[\mathrm{N} \mathrm{II}] / \mathrm{H} \alpha<1$.

\section{CONCLUSIONS}

We have studied the spectra of six low-activity nuclei with $[\mathrm{N}$ II $] / \mathrm{H} \alpha$ ratios larger than one. From the equivalent widths of the absorption lines we have selected one template spectrum representative of the stellar population of each nucleus. From previous results of spectral synthesis for these templates, we have concluded that the stellar population is mostly very old $\left(10^{10} \mathrm{yr}\right)$, and five of the observed galaxies have metal abundance higher than solar. We subtracted the stellar background from the spectra and measured the intensity of the emission lines by fitting Gaussians to the profiles. All the lines are broader than the instrumental profile. We have compared the relative ratios of the emission lines with values obtained from photoionization and shock models and found that our galaxies, as well as M51, M81, and M87, span a large range in nitrogen abundance from solar to 5 times solar. According to this, we suggest that the scatter in the diagrams involving the $[\mathrm{N} \mathrm{II}] / \mathrm{H} \alpha$ ratio of Seyfert 2 and LINER galaxies (for example, Terlevich and Melnick 1985) is due to this range in nitrogen abundance. It remains to be investigated if the overabundance of nitrogen in our galaxies is confined to the nucleus or is a general property of the whole galaxy.

We are grateful to the Cerro Tololo Inter-American Observatory where the observations and reductions were made. We also thank G. Ferland, S. M. Viegas-Aldrovandi, and R. B. Gruenwald for the photoionization models and D. Bica for the templates and fruitful discussions about the stellar population. M. G. P. is also grateful to the Royal Greenwich Observatory for its warm hospitality. and to B. Pagel, A. Diaz, and R. Terlevich for helpful comments. This work was partially supported by the Brazilian institutions FINEP and CNPq. 
Bica, E. 1988, Astr. Ap., 195, 76.

Bica, E., and Alloin, , D. 1986, Astr. Ap., 166, 83

Burbidge, E. M and Burbidge, G. R. 1962, Ap. J., 135, 694. 1965, Ap. J., 142, 634

Colbeck, L. A., ed. 1987, CTIO Facilities Manual

III Ferland, G., and Netzer, H. 1983, Ap. J., 264, 105.

Ferland, G., and Truran, J. W. 1980, Ap. J., 244, 1022

Filippenko, A. V., and Halpern, J. P. 1984, Ap. J., 285, 458

Filippenko, A. V., and Sargent, W. L. W. 1988, Ap. J., 324, 134

Gruenwald, R. B., and Pequignot, D. 1989, in IAU Symposium 131, Planetary Nebulae, ed. S. Torres-Peimbert (Dordrecht: Kluwer), p. 224

Heckman, T. M. $1980 a$, Astr. Ap., 87, 142.

. $1980 b$, Astr. Ap., 87, 152

$$
1980 b, \text { Astr. Ap., 87, } 152
$$

Keel, W. C. 1983a, Ap.J. Suppl., 52, 229.

$1983 b$, Ap. J., 269, 466

Pagel, B. E. J. 1983, in Formation and Evolution of Galaxies and Large Structures in the Universe, ed. J. Adouze and J. T. Thanh Van (Dordrecht: Reidel), p. 437.

Thaisa Storchi Bergmann and Miriani Griselda Pastoriza: Instituto de Fisica, Universidade Federal do Rio Grande do Sul,

Campus do Vale, Av. Bento Goncalves, 9500, Caixa Postal 15051, 91500 - Porto Alegre, RS, Brasil
Peimbert, M., and Torres-Peimbert, S. 1981, Ap. J., 245, 845.

Phillips, M. M., Charles, P. A., and Baldwin, J. A. 1983, Ap. J., 266, 485.

Rose, J. A., and Searle, L. 1982, Ap. J., 253, 556.

Rubin, V., and Ford, W. K., Jr. 1986, Ap. J.(Letters), 305, L35.

Sandage, A., Tammann, G. A. 1981, Revised Shapley-Ames Catalog of Bright Galaxies (Washington, DC: Carnegie Institution of Washington).

Seaton, M. J. 1979, M.N.R.A.S., 187, 73p.

Stasinska, G. 1984, Astr. Ap. Suppl., 55, 15.

Stauffer, J. R. 1982a, Ap. J. 262,66.

. 1982b, Ap.J. Suppl., 50, 517.

Stauffer, J. R., and Spinrad, H. 1979, Ap. J. (Letters), 231, L51.

Stone, R. P. S., and Baldwin, J. A. 1983, M.N.R.A.S., 204 347.

Terlevich, R., and Melnick, J. 1985, M.N.R.A.S., 213, 841.

Viegas-Aldrovandi, S. M. 1988, private communication.

Vigas-Aldrovandi, S. M., and Contini, M. 1989, Ap. J., in press.

Viegas-Aldrovandi, S. M., and Gruenwald, R. B. 1988, Ap. J., 324, 683. 